\title{
Ninety-Six Distinct Real Matrices for Representing a Quaternion Number
}

\author{
W. E. Ahmed (iD) 1,2 \\ ${ }^{1}$ Mathematics and Statistics Department, Faculty of Science, Imam Mohammad IBN Saud Islamic University, \\ Riyadh, Saudi Arabia \\ ${ }^{2}$ Department of Basics and Engineering Sciences, Faculty of Engineering, University of Khartoum, Khartoum, Sudan \\ Correspondence should be addressed to W. E. Ahmed; waahmed@imamu.edu.sa
}

Received 24 June 2020; Revised 30 September 2020; Accepted 28 October 2020; Published 4 December 2020

Academic Editor: Frank Uhlig

Copyright (C) 2020 W. E. Ahmed. This is an open access article distributed under the Creative Commons Attribution License, which permits unrestricted use, distribution, and reproduction in any medium, provided the original work is properly cited.

In this paper, we investigate on the number of all possible real matrices representing a quaternion number as three $4 \times 4$ skewsymmetric matrices plus the identity matrix of order 4 , and how to determine these matrices. We establish that there are 96 distinct real matrices having this property, and by matrix row operations, we obtain these matrices.

\section{Introduction}

Any quaternion number can be expressed in the form $a_{0}+a_{1} i_{1}+a_{2} i_{2}+a_{3} i_{3}$, where $a_{0}, a_{1}, a_{2}$, and $a_{3}$ are real numbers, and $i_{1}, i_{2}$, and $i_{3}$ are imaginary units which hold Hamilton's rule:

$$
\begin{aligned}
i_{1} i_{2} i_{3} & =-1, \\
i_{1}^{2} & =i_{2}^{2}=i_{3}^{2}=-1 .
\end{aligned}
$$

Table 1 gives all multiplications of these imaginary units. The quaternion multiplication is not commutative.

Quaternions have a great impact in developing mathematics $[1,2]$, for a review and to find out how quaternions were discovered by Sir William Rowan Hamilton, see [3-5] and [6] to take a general view on his life.

Quaternion numbers have representation as four-dimensional matrices over the real numbers [7], to see this, let

$$
a=a_{0}+a_{1} i_{1}+a_{2} i_{2}+a_{3} i_{3} .
$$

Multiply (2) from the right by $i_{1}, i_{2}$, and $i_{3}$, respectively,

$$
\begin{aligned}
& a \cdot i_{1}=a_{0} i_{1}-a_{1}-a_{2} i_{3}+a_{3} i_{2}, \\
& a \cdot i_{2}=a_{0} i_{2}+a_{1} i_{3}-a_{2}-a_{3} i_{1}, \\
& a \cdot i_{3}=a_{0} i_{3}-a_{1} i_{2}+a_{2} i_{1}-a_{3} .
\end{aligned}
$$

We can make a matrix of the coefficients using the basis $\left\{1, i_{1}, i_{2}, i_{3}\right\}$ as follows:

$$
\begin{aligned}
& A_{1}=\left[\begin{array}{cccc}
a_{0} & -a_{1} & -a_{2} & -a_{3} \\
a_{1} & a_{0} & -a_{3} & a_{2} \\
a_{2} & a_{3} & a_{0} & -a_{1} \\
a_{3} & -a_{2} & a_{1} & a_{0}
\end{array}\right]=a_{0}\left[\begin{array}{cccc}
1 & 0 & 0 & 0 \\
0 & 1 & 0 & 0 \\
0 & 0 & 1 & 0 \\
0 & 0 & 0 & 1
\end{array}\right]+a_{1}\left[\begin{array}{cccc}
0 & -1 & 0 & 0 \\
1 & 0 & 0 & 0 \\
0 & 0 & 0 & -1 \\
0 & 0 & 1 & 0
\end{array}\right] \\
& +a_{2}\left[\begin{array}{cccc}
0 & 0 & -1 & 0 \\
0 & 0 & 0 & 1 \\
1 & 0 & 0 & 0 \\
0 & -1 & 0 & 0
\end{array}\right]+a_{3}\left[\begin{array}{cccc}
0 & 0 & 0 & -1 \\
0 & 0 & -1 & 0 \\
0 & 1 & 0 & 0 \\
1 & 0 & 0 & 0
\end{array}\right] \\
& =a_{0} I_{4}+a_{1} M_{1}+a_{2} M_{2}+a_{3} M_{3} \text {. }
\end{aligned}
$$

$I_{4}$ is the identity, and $M_{1}, M_{2}$, and $M_{3}$ have the properties:

$$
\begin{aligned}
M_{i}^{T} & =-M_{i}, \\
M_{i}^{T} M_{i} & =I, \\
M_{i} M_{i} & =-I, \quad \text { where } M_{i}^{T} \text { is transpose of } M_{i}, i=1,2,3 .
\end{aligned}
$$


TABLE 1: Imaginary units multiplication.

\begin{tabular}{cccc}
\hline & $i_{1}$ & $i_{2}$ & $i_{3}$ \\
\hline$i_{1}$ & -1 & $i_{3}$ & $-i_{2}$ \\
$i_{2}$ & $-i_{3}$ & -1 & $i_{1}$ \\
$i_{3}$ & $i_{2}$ & $-i_{1}$ & -1 \\
\hline
\end{tabular}

A square matrix whose transpose equals its negative is said to be skew-symmetric (or antimetric) [8], $M_{1}, M_{2}$, and $M_{3}$ are $4 \times 4$ skew-symmetric matrices.

From the basic properties of transposes [9], we know that if $M$ is skew-symmetric matrix, then the transpose $M^{T}$ is skew-symmetric too, so the matrix representation of a quaternion number is not unique.

The transpose matrix of $A_{1}$ gives another representation of a quaternion number $a$ :

$$
\begin{aligned}
B_{1}=A_{1}^{T}=\left[\begin{array}{cccc}
a_{0} & a_{1} & a_{2} & a_{3} \\
-a_{1} & a_{0} & a_{3} & -a_{2} \\
-a_{2} & -a_{3} & a_{0} & a_{1} \\
-a_{3} & a_{2} & -a_{1} & a_{0}
\end{array}\right] \\
=a_{0}\left[\begin{array}{llll}
0 & 1 & 0 & 0 \\
0 & 0 & 1 & 0 \\
0 & 0 & 0 & 1
\end{array}\right]+a_{1}\left[\begin{array}{cccc}
0 & 1 & 0 & 0 \\
-1 & 0 & 0 & 0 \\
0 & 0 & 0 & -1 \\
0 & 0 & 1 & 0
\end{array}\right] \\
+a_{2}\left[\begin{array}{cccc}
0 & 0 & 1 & 0 \\
0 & 0 & 0 & -1 \\
-1 & 0 & 0 & 0 \\
0 & 1 & 0 & 0
\end{array}\right]+a_{3}\left[\begin{array}{cccc}
0 & 0 & 0 & 1 \\
0 & 0 & 1 & 0 \\
0 & -1 & 0 & 0 \\
-1 & 0 & 0 & 0
\end{array}\right] \\
=a_{0} I_{4}+a_{1} N_{1}+a_{2} N_{2}+a_{3} N_{3} . \\
\end{aligned}
$$

$N_{1}, N_{2}$, and $N_{3}$ are $4 \times 4$ skew-symmetric matrices.

The purpose of this paper is to eliminate the confusion that existing about the total number of real matrices that can represent a quaternion number.

We investigate the questions:

(1) How many distinct matrices in the form of $A_{1}$ can give representation for a quaternion number?

(2) How to determine these matrices?

We will consider two sets of matrices:
Left matrix representation and its transpose

Right matrix representation and its transpose

Farebrother et al. [10] take one of these two sets and talk about 48 distinct matrices representation for a quaternion number.

\section{Discussion and Results}

Let us split the matrix $A_{1}$ into four $2 \times 2$ matrices; of course each matrix has an element takes a different sign from the others:

$$
A_{1}=\left[\begin{array}{cc}
\varphi_{1}(a) & -\varphi_{2}(b) \\
\varphi_{2}(b) & \varphi_{1}(a)
\end{array}\right]
$$

where $\varphi_{1}(a)=\left[\begin{array}{cc}a_{0} & -a_{1} \\ a_{1} & a_{0}\end{array}\right]$ and $\varphi_{2}(b)=\left[\begin{array}{cc}a_{2} & a_{3} \\ a_{3} & -a_{2}\end{array}\right]$.

$\varphi_{1}$ has 6 forms:

$$
\begin{aligned}
& {\left[\begin{array}{ll}
a_{0} & -a_{1} \\
a_{1} & a_{0}
\end{array}\right],\left[\begin{array}{cc}
a_{0} & a_{1} \\
-a_{1} & a_{0}
\end{array}\right],\left[\begin{array}{ll}
a_{0} & -a_{2} \\
a_{2} & a_{0}
\end{array}\right],\left[\begin{array}{cc}
a_{0} & a_{2} \\
-a_{2} & a_{0}
\end{array}\right],} \\
& {\left[\begin{array}{ll}
a_{0} & -a_{3} \\
a_{3} & a_{0}
\end{array}\right],\left[\begin{array}{ll}
a_{0} & a_{3} \\
-a_{3} & a_{0}
\end{array}\right],}
\end{aligned}
$$

whereas $\varphi_{2}$ (two elements remaining, say $a_{2}$ and $a_{3}$ ) has 16 forms:

$$
\begin{aligned}
& {\left[\begin{array}{cc}
a_{2} & a_{3} \\
a_{3} & -a_{2}
\end{array}\right],\left[\begin{array}{ll}
-a_{2} & a_{3} \\
a_{3} & a_{2}
\end{array}\right],\left[\begin{array}{ll}
a_{2} & -a_{3} \\
a_{3} & a_{2}
\end{array}\right],\left[\begin{array}{cc}
a_{2} & a_{3} \\
-a_{3} & a_{2}
\end{array}\right],} \\
& {\left[\begin{array}{ll}
a_{2} & -a_{3} \\
-a_{3} & -a_{2}
\end{array}\right],\left[\begin{array}{ll}
-a_{2} & -a_{3} \\
-a_{3} & a_{2}
\end{array}\right],\left[\begin{array}{ll}
-a_{2} & a_{3} \\
-a_{3} & -a_{2}
\end{array}\right],\left[\begin{array}{ll}
-a_{2} & -a_{3} \\
a_{3} & -a_{2}
\end{array}\right],}
\end{aligned}
$$$$
\left[\begin{array}{cc}
a_{3} & a_{2} \\
a_{2} & -a_{3}
\end{array}\right],\left[\begin{array}{cc}
-a_{3} & a_{2} \\
a_{2} & a_{3}
\end{array}\right],\left[\begin{array}{cc}
a_{3} & -a_{2} \\
a_{2} & a_{3}
\end{array}\right],\left[\begin{array}{cc}
a_{3} & a_{2} \\
-a_{2} & a_{3}
\end{array}\right],
$$

$\left[\begin{array}{cc}a_{3} & -a_{2} \\ -a_{2} & -a_{3}\end{array}\right],\left[\begin{array}{cc}-a_{3} & -a_{2} \\ -a_{2} & a_{3}\end{array}\right],\left[\begin{array}{cc}-a_{3} & a_{2} \\ -a_{2} & -a_{3}\end{array}\right],\left[\begin{array}{cc}-a_{3} & -a_{2} \\ a_{2} & -a_{3}\end{array}\right]$.

Therefore, the total number of all possible matrices is (6) $(16)=96$.

To determine these matrices, we do some operations on both columns and rows of the matrix $A_{1}$ (Tables 2-4 and Algorithms 1-6). We will find 
TABle 2: One switching operation.

\begin{tabular}{lrr}
\hline Operation & Model & Number of all possible matrices \\
\hline (1) One switching: selecting two columns to switch them together & $* \overbrace{*}$ & $4 ! / 2 ! 2 !=6$ \\
\hline
\end{tabular}

TABle 3: Two switching operations.

Operation
(2) Two switching:
(i) Selecting two columns to switch them, and switching the other two columns together
(ii) Selecting three columns then selecting one of them to switch with the others

TABLE 4: Three switching operations.

(3) Three switching: selecting a column to switch with a second column, then switching this second column with a third column, and switching this third column

\#Python code for one column (row) switching

def OneSwitching $(A, n, m)$ :

for $i$ in range (4):

$R=A[n][i]$

$A[n][i]=A[m][i]$

$A[m][i]=R$

for $j$ in range (4):

$C=A[j][n]$

$A[j][n]=A[j][m]$

$A[j][m]=C$

$m=1$ \# Replacing $m$ by 2 and another time by 3 gives all 6 matrices

for $n$ in range $(m)$ :

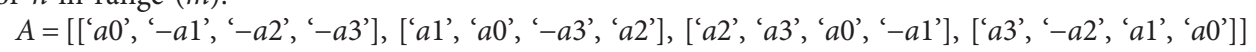

print (“The Matrix $=n$ ")

OneSwitching $(A, n, m)$

for $i$ in range (4):

for $j$ in range (4):

print $(A[i][j]$, end $=$ “”)

print (“\n")

Algorithm 1: One switching 1.

\#Python code for two columns (rows) switching

def TwoSwitching1 $(A, n, m)$ :

for $i$ in range (4):

$R 1=A[0][i]$

$A[0][i]=A[2 * m-n-1][i]$

$A[2 * m-n-1][i]=R 1$

$R 2=A[2 * k+m-1][i]$

$A[2 * k+m-1][i]=A[2 * k+m+n][i]$

$A[2 * k+m+n][i]=R 2$

for $j$ in range (4):

$C 1=A[j][0]$

$A[j][0]=A[j][2 * m-n-1]$

$A[j][2 * m-n-1]=C 1$

$C 2=A[j][2 * k+m-1]$

$A[j][2 * k+m-1]=A[j][2 * k+m+n]$ 
$m=2$

$A[j][2 * k+m+n]=C 2$

$k=0 \#$ another time put $m=1$ and $k=1$

for $n$ in range $(m)$ :

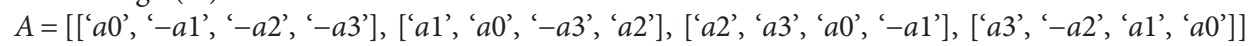
print ("The Matrix $=n$ ")

TwoSwitching1 $(A, n, m)$

for $i$ in range (4):

for $j$ in range (4):

print $(A[i][j]$, end $=$ “”)

print (" $\backslash n$ ")

Algorithm 2: Two switching operations 1.

\#Python code for two columns (rows) switching

def TwoSwitching2 $(A, n, m)$ :

for $i$ in range (4):

$R=A[0][i]$

$A[0][i]=A[2 * k+1][i]$

$A[2 * k+1][i]=A[n+m-k][i]$

$A[n+m-k][i]=R$

for $j$ in range (4):

$C=A[j][0]$

$A[j][0]=A[j][2 * k+1]$

$A[j][2 * k+1]=A[j][n+m-k]$

$m=2$

$A[j][n+m-k]=C$

$k=0 \#$ another time put $k=1$

for $n$ in range $(m)$ :

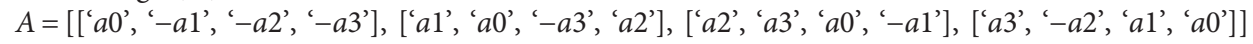
print ("The Matrix $=n$ ")

TwoSwitching2 $(A, n, m)$

for $i$ in range (4):

for $j$ in range (4):

print $(A[i][j]$, end $=$ " ”)

print (" $\backslash n$ ")

Algorithm 3: Two switching 2.

\#Python code for two columns (rows) switching

def TwoSwitching3 $(A, n, m)$ :

for $i$ in range (4):

$R=A[n][i]$

$A[n][i]=A[k * n+m][i]$

$A[k * n+m][i]=A[k *(n-2)+m+1][i]$

$A[k *(n-2)+m+1][i]=R$

for $j$ in range (4):

$C=A[j][n]$

$A[j][n]=A[j][k * n+m]$

$A[j][k * n+m]=A[j][k *(n-2)+m+1]$

$m=2$

$A[j][k *(n-2)+m+1]=C$

$k=0 \#$ another time put $k=1$

for $n$ in range $(m)$ :

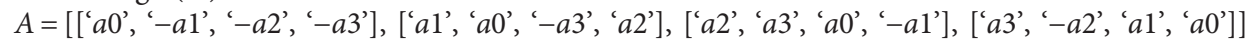
print ("The Matrix $=n$ ") 
TwoSwitching3 $(A, n, m)$

for $i$ in range (4):

for $j$ in range (4):

print $(A[i][j]$, end $=$ " ”)

print (" $\backslash n$ ")

Algorithm 4: Two switching 3.

\#Python code for three columns (rows) switching

def ThreeSwitching1 $(A, n, m)$ :

for $i$ in range (4):

$R=A[0][i]$

$A[0][i]=A[2 * k+1][i]$

$A[2 * k+1][i]=A[n+m-k][i]$

$A[n+m-k][i]=A[2 * m-n-k-1][i]$

$A[2 * m-n-k-1][i]=R$

for $j$ in range (4):

$C=A[j][0]$

$A[j][0]=A[j][2 * k+1]$

$A[j][2 * k+1]=A[j][n+m-k]$

$A[j][n+m-k]=A[j][2 * m-n-k-1]$

$m=2$

$A[j][2 * m-n-k-1]=C$

$k=0 \#$ another time $k=1$

for $n$ in range $(\mathrm{m})$ :

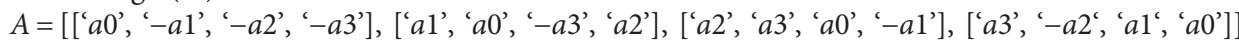

print ("The Matrix $=n$ ")

ThreeSwitching1 $(A, n, m)$

for $i$ in range (4):

for $j$ in range(4):

print $(A[i][j]$, end $=$ “”)

print (“\n")

Algorithm 5: Three switching 1.

\#Python code for three columns (rows) switching

def ThreeSwitching2 $(A, n, m)$ :

for $i$ in range (4):

$R=A[n][i]$

$A[n][i]=A[m-2 * n][i]$

$A[m-2 * n][i]=A[n+m-1][i]$

$A[n+m-1][i]=A[m+1][i]$

$A[m+1][i]=R$

for $j$ in range (4):

$C=A[j][n]$

$A[j][n]=A[j][m-2 * n]$

$A[j][m-2 * n]=A[j][n+m-1]$

$A[j][n+m-1]=A[j][m+1]$

$A[j][m+1]=C$

$m=2$

for $n$ in range $(\mathrm{m})$ :

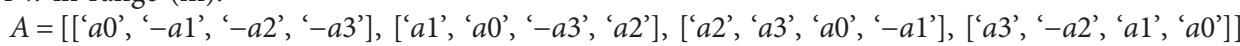
print ("The Matrix $=n$ ")

ThreeSwitching2 $(A, n, m)$

for $i$ in range (4):

for $j$ in range (4):

print $(A[i][j]$, end $=$ " ”)

print (" $\mid n$ ")

Algorithm 6: Three switching 2. 


$$
\begin{aligned}
& C_{1} \longleftrightarrow C_{2}, R_{1} \longleftrightarrow R_{2}, \\
& A_{2}=\left[\begin{array}{cccc}
a_{0} & a_{1} & -a_{3} & a_{2} \\
-a_{1} & a_{0} & -a_{2} & -a_{3} \\
a_{3} & a_{2} & a_{0} & -a_{1} \\
-a_{2} & a_{3} & a_{1} & a_{0}
\end{array}\right], \\
& C_{1} \longleftrightarrow C_{3}, R_{1} \longleftrightarrow R_{3} \text {, } \\
& A_{3}=\left[\begin{array}{cccc}
a_{0} & a_{3} & a_{2} & -a_{1} \\
-a_{3} & a_{0} & a_{1} & a_{2} \\
-a_{2} & -a_{1} & a_{0} & -a_{3} \\
a_{1} & -a_{2} & a_{3} & a_{0}
\end{array}\right], \\
& C_{1} \longleftrightarrow C_{4}, R_{1} \longleftrightarrow R_{4} \text {, } \\
& A_{4}=\left[\begin{array}{cccc}
a_{0} & -a_{2} & a_{1} & a_{3} \\
a_{2} & a_{0} & -a_{3} & a_{1} \\
-a_{1} & a_{3} & a_{0} & a_{2} \\
-a_{3} & -a_{1} & -a_{2} & a_{0}
\end{array}\right], \\
& C_{2} \longleftrightarrow C_{3}, R_{2} \longleftrightarrow R_{3} \text {, } \\
& A_{5}=\left[\begin{array}{cccc}
a_{0} & -a_{2} & -a_{1} & -a_{3} \\
a_{2} & a_{0} & a_{3} & -a_{1} \\
a_{1} & -a_{3} & a_{0} & a_{2} \\
a_{3} & a_{1} & -a_{2} & a_{0}
\end{array}\right], \\
& \mathrm{C}_{2} \longleftrightarrow \mathrm{C}_{4}, \mathrm{R}_{2} \longleftrightarrow \mathrm{R}_{4} \text {, } \\
& A_{6}=\left[\begin{array}{cccc}
a_{0} & -a_{3} & -a_{2} & -a_{1} \\
a_{3} & a_{0} & a_{1} & -a_{2} \\
a_{2} & -a_{1} & a_{0} & a_{3} \\
a_{1} & a_{2} & -a_{3} & a_{0}
\end{array}\right], \\
& C_{3} \longleftrightarrow C_{4}, R_{3} \longleftrightarrow R_{4} \text {, } \\
& A_{7}=\left[\begin{array}{cccc}
a_{0} & -a_{1} & -a_{3} & -a_{2} \\
a_{1} & a_{0} & a_{2} & -a_{3} \\
a_{3} & -a_{2} & a_{0} & a_{1} \\
a_{2} & a_{3} & -a_{1} & a_{0}
\end{array}\right], \\
& C_{1} \longleftrightarrow C_{2}, C_{3} \longleftrightarrow C_{4}, \\
& R_{1} \longleftrightarrow R_{2}, R_{3} \longleftrightarrow R_{4} \text {, } \\
& A_{8}=\left[\begin{array}{cccc}
a_{0} & a_{1} & a_{2} & -a_{3} \\
-a_{1} & a_{0} & -a_{3} & -a_{2} \\
-a_{2} & a_{3} & a_{0} & a_{1} \\
a_{3} & a_{2} & -a_{1} & a_{0}
\end{array}\right], \\
& C_{1} \longleftrightarrow C_{3}, C_{2} \longleftrightarrow C_{4} \text {, } \\
& R_{1} \longleftrightarrow R_{3}, R_{2} \longleftrightarrow R_{4} \text {, } \\
& A_{9}=\left[\begin{array}{cccc}
a_{0} & -a_{1} & a_{2} & a_{3} \\
a_{1} & a_{0} & a_{3} & -a_{2} \\
-a_{2} & -a_{3} & a_{0} & -a_{1} \\
-a_{3} & a_{2} & a_{1} & a_{0}
\end{array}\right], \\
& C_{1} \longleftrightarrow C_{4}, C_{2} \longleftrightarrow C_{3} \text {, } \\
& R_{1} \longleftrightarrow R_{4}, R_{2} \longleftrightarrow R_{3} \text {, } \\
& A_{10}=\left[\begin{array}{cccc}
a_{0} & a_{1} & -a_{2} & a_{3} \\
-a_{1} & a_{0} & a_{3} & a_{2} \\
a_{2} & -a_{3} & a_{0} & a_{1} \\
-a_{3} & -a_{2} & -a_{1} & a_{0}
\end{array}\right],
\end{aligned}
$$

$$
\begin{aligned}
& C_{1} \longleftrightarrow C_{2} \longleftrightarrow C_{3}, \\
& R_{1} \longleftrightarrow R_{2} \longleftrightarrow R_{3} \text {, } \\
& A_{11}=\left[\begin{array}{cccc}
a_{0} & a_{2} & a_{3} & -a_{1} \\
-a_{2} & a_{0} & -a_{1} & -a_{3} \\
-a_{3} & a_{1} & a_{0} & a_{2} \\
a_{1} & a_{3} & -a_{2} & a_{0}
\end{array}\right], \\
& C_{1} \longleftrightarrow C_{2} \longleftrightarrow C_{4} \text {, } \\
& R_{1} \longleftrightarrow R_{2} \longleftrightarrow R_{4} \text {, } \\
& A_{12}=\left[\begin{array}{cccc}
a_{0} & a_{3} & a_{1} & -a_{2} \\
-a_{3} & a_{0} & -a_{2} & -a_{1} \\
-a_{1} & a_{2} & a_{0} & a_{3} \\
a_{2} & a_{1} & -a_{3} & a_{0}
\end{array}\right], \\
& C_{1} \longleftrightarrow C_{4} \longleftrightarrow C_{2} \text {, } \\
& R_{1} \longleftrightarrow R_{4} \longleftrightarrow R_{2} \text {, } \\
& A_{13}=\left[\begin{array}{cccc}
a_{0} & a_{2} & -a_{3} & a_{1} \\
-a_{2} & a_{0} & a_{1} & a_{3} \\
a_{3} & -a_{1} & a_{0} & a_{2} \\
-a_{1} & -a_{3} & -a_{2} & a_{0}
\end{array}\right], \\
& C_{1} \longleftrightarrow C_{4} \longleftrightarrow C_{3} \text {, } \\
& R_{1} \longleftrightarrow R_{4} \longleftrightarrow R_{3} \text {, } \\
& A_{14}=\left[\begin{array}{cccc}
a_{0} & a_{3} & -a_{1} & a_{2} \\
-a_{3} & a_{0} & a_{2} & a_{1} \\
a_{1} & -a_{2} & a_{0} & a_{3} \\
-a_{2} & -a_{1} & -a_{3} & a_{0}
\end{array}\right], \\
& C_{1} \longleftrightarrow C_{3} \longleftrightarrow C_{4} \text {, } \\
& R_{1} \longleftrightarrow R_{3} \longleftrightarrow R_{4} \text {, } \\
& A_{15}=\left[\begin{array}{cccc}
a_{0} & -a_{2} & a_{3} & a_{1} \\
a_{2} & a_{0} & a_{1} & -a_{3} \\
-a_{3} & -a_{1} & a_{0} & -a_{2} \\
-a_{1} & a_{3} & a_{2} & a_{0}
\end{array}\right], \\
& C_{2} \longleftrightarrow C_{3} \longleftrightarrow C_{4} \text {, } \\
& R_{2} \longleftrightarrow R_{3} \longleftrightarrow R_{4}, \\
& A_{16}=\left[\begin{array}{cccc}
a_{0} & -a_{3} & -a_{1} & -a_{2} \\
a_{3} & a_{0} & -a_{2} & a_{1} \\
a_{1} & a_{2} & a_{0} & -a_{3} \\
a_{2} & -a_{1} & a_{3} & a_{0}
\end{array}\right], \\
& C_{1} \longleftrightarrow C_{3} \longleftrightarrow C_{2} \text {, } \\
& R_{1} \longleftrightarrow R_{3} \longleftrightarrow R_{2} \text {, } \\
& A_{17}=\left[\begin{array}{cccc}
a_{0} & -a_{3} & a_{1} & a_{2} \\
a_{3} & a_{0} & a_{2} & -a_{1} \\
-a_{1} & -a_{2} & a_{0} & -a_{3} \\
-a_{2} & a_{1} & a_{3} & a_{0}
\end{array}\right], \\
& \mathrm{C}_{2} \longleftrightarrow \mathrm{C}_{4} \longleftrightarrow \mathrm{C}_{3} \text {, } \\
& R_{2} \longleftrightarrow R_{4} \longleftrightarrow R_{3}, \\
& A_{18}=\left[\begin{array}{cccc}
a_{0} & -a_{2} & -a_{3} & a_{1} \\
a_{2} & a_{0} & -a_{1} & a_{3} \\
a_{3} & a_{1} & a_{0} & -a_{2} \\
a_{1} & -a_{3} & a_{2} & a_{0}
\end{array}\right],
\end{aligned}
$$




$$
\begin{aligned}
& C_{1} \longleftrightarrow C_{2} \longleftrightarrow C_{3} \longleftrightarrow C_{4}, \\
& R_{1} \longleftrightarrow R_{2} \longleftrightarrow R_{3} \longleftrightarrow R_{4}, \\
& A_{19}=\left[\begin{array}{cccc}
a_{0} & -a_{3} & a_{2} & a_{1} \\
a_{3} & a_{0} & -a_{1} & a_{2} \\
-a_{2} & a_{1} & a_{0} & a_{3} \\
-a_{1} & -a_{2} & -a_{3} & a_{0}
\end{array}\right], \\
& C_{1} \longleftrightarrow C_{2} \longleftrightarrow C_{4} \longleftrightarrow C_{3}, \\
& R_{1} \longleftrightarrow R_{2} \longleftrightarrow R_{4} \longleftrightarrow R_{3}, \\
& A_{20}=\left[\begin{array}{cccc}
a_{0} & a_{2} & a_{1} & -a_{3} \\
-a_{2} & a_{0} & a_{3} & a_{1} \\
-a_{1} & -a_{3} & a_{0} & -a_{2} \\
a_{3} & -a_{1} & a_{2} & a_{0}
\end{array}\right], \\
& C_{1} \longleftrightarrow C_{4} \longleftrightarrow C_{2} \longleftrightarrow C_{3}, \\
& R_{1} \longleftrightarrow R_{4} \longleftrightarrow R_{2} \longleftrightarrow R_{3}, \\
& A_{21}=\left[\begin{array}{cccc}
a_{0} & a_{3} & -a_{2} & a_{1} \\
-a_{3} & a_{0} & -a_{1} & -a_{2} \\
a_{2} & a_{1} & a_{0} & -a_{3} \\
-a_{1} & a_{2} & a_{3} & a_{0}
\end{array}\right], \\
& C_{1} \longleftrightarrow C_{4} \longleftrightarrow C_{3} \longleftrightarrow C_{2}, \\
& R_{1} \longleftrightarrow R_{4} \longleftrightarrow R_{3} \longleftrightarrow R_{2}, \\
& A_{22}=\left[\begin{array}{cccc}
a_{0} & a_{1} & a_{3} & -a_{2} \\
-a_{1} & a_{0} & a_{2} & a_{3} \\
-a_{3} & -a_{2} & a_{0} & -a_{1} \\
a_{2} & -a_{3} & a_{1} & a_{0}
\end{array}\right],
\end{aligned}
$$

$$
\begin{aligned}
& C_{1} \longleftrightarrow C_{3} \longleftrightarrow C_{2} \longleftrightarrow C_{4}, \\
& R_{1} \longleftrightarrow R_{3} \longleftrightarrow R_{2} \longleftrightarrow R_{4}, \\
& A_{23}=\left[\begin{array}{cccc}
a_{0} & -a_{1} & a_{3} & a_{2} \\
a_{1} & a_{0} & -a_{2} & a_{3} \\
-a_{3} & a_{2} & a_{0} & a_{1} \\
-a_{2} & -a_{3} & -a_{1} & a_{0}
\end{array}\right], \\
& C_{2} \longleftrightarrow C_{1} \longleftrightarrow C_{3} \longleftrightarrow C_{4}, \\
& R_{2} \longleftrightarrow R_{1} \longleftrightarrow R_{3} \longleftrightarrow R_{4}, \\
& A_{24}=\left[\begin{array}{cccc}
a_{0} & a_{2} & -a_{1} & a_{3} \\
-a_{2} & a_{0} & -a_{3} & -a_{1} \\
a_{1} & a_{3} & a_{0} & -a_{2} \\
-a_{3} & a_{1} & a_{2} & a_{0}
\end{array}\right] .
\end{aligned}
$$

We obtained 23 distinct matrices from the matrix $A_{1}$, and by similar way, we can obtain another 23 matrices from the matrix $B_{1}$.

Also, we can get another 46 matrices from $C_{1}$ and $D_{1}$.

We stablish $C_{1}$ by multiplying (2) from the left by $i_{1}, i_{2}$, and $i_{3}$, respectively,

$$
\begin{aligned}
& i_{1} \cdot a=a_{0} i_{1}-a_{1}+a_{2} i_{3}-a_{3} i_{2}, \\
& i_{2} \cdot a=a_{0} i_{2}-a_{1} i_{3}-a_{2}+a_{3} i_{1}, \\
& i_{3} \cdot a=a_{0} i_{3}+a_{1} i_{2}-a_{2} i_{1}-a_{3} .
\end{aligned}
$$

So,

$$
\begin{aligned}
C_{1}= & {\left[\begin{array}{cccc}
a_{0} & -a_{1} & -a_{2} & -a_{3} \\
a_{1} & a_{0} & a_{3} & -a_{2} \\
a_{2} & -a_{3} & a_{0} & a_{1} \\
a_{3} & a_{2} & -a_{1} & a_{0}
\end{array}\right]=a_{0}\left[\begin{array}{llll}
1 & 0 & 0 & 0 \\
0 & 1 & 0 & 0 \\
0 & 0 & 1 & 0 \\
0 & 0 & 0 & 1
\end{array}\right]+a_{1}\left[\begin{array}{cccc}
0 & -1 & 0 & 0 \\
1 & 0 & 0 & 0 \\
0 & 0 & 0 & -1 \\
0 & 0 & 1 & 0
\end{array}\right] } \\
& +a_{2}\left[\begin{array}{cccc}
0 & 0 & -1 & 0 \\
0 & 0 & 0 & -1 \\
1 & 0 & 0 & 0 \\
0 & 1 & 0 & 0
\end{array}\right]+a_{3}\left[\begin{array}{cccc}
0 & 0 & 0 & -1 \\
0 & 0 & 1 & 0 \\
0 & -1 & 0 & 0 \\
1 & 0 & 0 & 0
\end{array}\right]=a_{0} I_{4}+a_{1} S_{1}+a_{2} S_{2}+a_{3} S_{3} .
\end{aligned}
$$


$S_{1}, S_{2}$, and $S_{3}$ are $4 \times 4$ skew-symmetric matrices, whereas $D_{1}$ is the transpose of $C_{1}$ :

$$
\begin{aligned}
D_{1}= & C_{1}^{T}=\left[\begin{array}{cccc}
a_{0} & a_{1} & a_{2} & a_{3} \\
-a_{1} & a_{0} & -a_{3} & a_{2} \\
-a_{2} & a_{3} & a_{0} & -a_{1} \\
-a_{3} & -a_{2} & a_{1} & a_{0}
\end{array}\right]=a_{0}\left[\begin{array}{cccc}
1 & 0 & 0 & 0 \\
0 & 1 & 0 & 0 \\
0 & 0 & 1 & 0 \\
0 & 0 & 0 & 1
\end{array}\right]+a_{1}\left[\begin{array}{cccc}
0 & 1 & 0 & 0 \\
-1 & 0 & 0 & 0 \\
0 & 0 & 0 & -1 \\
0 & 0 & 1 & 0
\end{array}\right] \\
& +a_{2}\left[\begin{array}{cccc}
0 & 0 & 1 & 0 \\
0 & 0 & 0 & 1 \\
-1 & 0 & 0 & 0 \\
0 & -1 & 0 & 0
\end{array}\right]+a_{3}\left[\begin{array}{cccc}
0 & 0 & 0 & 1 \\
0 & 0 & -1 & 0 \\
0 & 1 & 0 & 0 \\
-1 & 0 & 0 & 0
\end{array}\right]=a_{0} I_{4}+a_{1} T_{1}+a_{2} T_{2}+a_{3} T_{3} .
\end{aligned}
$$

$T_{1}, T_{2}$, and $T_{3}$ are $4 \times 4$ skew-symmetric matrices.

We put these 96 matrices into two sets:

Left matrices representation set which consists of $A_{\mathrm{i}}$ and $D_{i}$

Right matrices representation set which consists of $B_{\mathrm{i}}$ and $C_{\mathrm{i}}$ where $(1 \leq i \leq 24)$
In solving quaternions problems, we have to pay attention to the difference between the two sets, for example, if we want to find the product $a \mathrm{~b}$, where $a=a_{0}+a_{1} i_{1}+a_{2} i_{2}+$ $a_{3} i_{3}$ and $b=b_{0}+b_{1} i_{1}+b_{2} i_{2}+b_{3} i_{3}$.

(1) By using left matrices representation:

If we take $A_{24}$ (for example),

$$
\left[\begin{array}{cccc}
a_{0} & a_{2} & -a_{1} & a_{3} \\
-a_{2} & a_{0} & -a_{3} & -a_{1} \\
a_{1} & a_{3} & a_{0} & -a_{2} \\
-a_{3} & a_{1} & a_{2} & a_{0}
\end{array}\right]\left[\begin{array}{c}
b_{0} \\
-b_{2} \\
b_{1} \\
-b_{3}
\end{array}\right]=\left[\begin{array}{c}
a_{0} b_{0}-a_{2} b_{2}-a_{1} b_{1}-a_{3} b_{3} \\
-a_{2} b_{0}-a_{0} b_{2}-a_{3} b_{1}+a_{1} b_{3} \\
a_{1} b_{0}-a_{3} b_{2}+a_{0} b_{1}+a_{2} b_{3} \\
-a_{3} b_{0}-a_{1} b_{2}+a_{2} b_{1}-a_{0} b_{3}
\end{array}\right] .
$$

Thus,

$$
\begin{aligned}
a \mathrm{~b}= & \left(a_{0} b_{0}-a_{2} b_{2}-a_{1} b_{1}-a_{3} b_{3}\right)+\left(a_{1} b_{0}-a_{3} b_{2}+a_{0} b_{1}+a_{2} b_{3}\right) i_{1} \\
& -\left(-a_{2} b_{0}-a_{0} b_{2}-a_{3} b_{1}+a_{1} b_{3}\right) i_{2}-\left(-a_{3} b_{0}-a_{1} b_{2}+a_{2} b_{1}-a_{0} b_{3}\right) i_{3} .
\end{aligned}
$$

If we take $D_{1}$,

$$
\left[\begin{array}{cccc}
a_{0} & a_{1} & a_{2} & a_{3} \\
-a_{1} & a_{0} & -a_{3} & a_{2} \\
-a_{2} & a_{3} & a_{0} & -a_{1} \\
-a_{3} & -a_{2} & a_{1} & a_{0}
\end{array}\right]\left[\begin{array}{c}
b_{0} \\
-b_{1} \\
-b_{2} \\
-b_{3}
\end{array}\right]=\left[\begin{array}{c}
a_{0} b_{0}-a_{1} b_{1}-a_{2} b_{2}-a_{3} b_{3} \\
-a_{1} b_{0}-a_{0} b_{1}+a_{3} b_{2}-a_{2} b_{3} \\
-a_{2} b_{0}-a_{3} b_{1}-a_{0} b_{2}+a_{1} b_{3} \\
-a_{3} b_{0}+a_{2} b_{1}-a_{1} b_{2}-a_{0} b_{3}
\end{array}\right] .
$$

Thus,

$$
\begin{aligned}
a \mathrm{~b}= & \left(a_{0} b_{0}-a_{1} b_{1}-a_{2} b_{2}-a_{3} b_{3}\right)-\left(-a_{1} b_{0}-a_{0} b_{1}+a_{3} b_{2}-a_{2} b_{3}\right) i_{1} \\
& -\left(-a_{2} b_{0}-a_{3} b_{1}-a_{0} b_{2}+a_{1} b_{3}\right) i_{2}-\left(-a_{3} b_{0}+a_{2} b_{1}-a_{1} b_{2}-a_{0} b_{3}\right) i_{3} .
\end{aligned}
$$


(2) By using right matrices representation:

If we take $B_{1}$,

$$
\left[\begin{array}{cccc}
b_{0} & b_{1} & b_{2} & b_{3} \\
-b_{1} & b_{0} & b_{3} & -b_{2} \\
-b_{2} & -b_{3} & b_{0} & b_{1} \\
-b_{3} & b_{2} & -b_{1} & b_{0}
\end{array}\right]\left[\begin{array}{c}
a_{0} \\
-a_{1} \\
-a_{2} \\
-a_{3}
\end{array}\right]=\left[\begin{array}{l}
a_{0} b_{0}-a_{1} b_{1}-a_{2} b_{2}-a_{3} b_{3} \\
-a_{0} b_{1}-a_{1} b_{0}-a_{2} b_{3}+a_{3} b_{2} \\
-a_{0} b_{2}+a_{1} b_{3}-a_{2} b_{0}-a_{3} b_{1} \\
-a_{0} b_{3}-a_{1} b_{2}+a_{2} b_{1}-a_{3} b_{0}
\end{array}\right] .
$$

Thus,

$$
\begin{aligned}
a \mathrm{~b}= & \left(a_{0} b_{0}-a_{1} b_{1}-a_{2} b_{2}-a_{3} b_{3}\right)-\left(-a_{0} b_{1}-a_{1} b_{0}-a_{2} b_{3}+a_{3} b_{2}\right) i_{1} \\
& -\left(-a_{0} b_{2}+a_{1} b_{3}-a_{2} b_{0}-a_{3} b_{1}\right) i_{2}-\left(-a_{0} b_{3}-a_{1} b_{2}+a_{2} b_{1}-a_{3} b_{0}\right) i_{3} .
\end{aligned}
$$

If we take $C_{1}$,

$$
\left[\begin{array}{cccc}
b_{0} & -b_{1} & -b_{2} & -b_{3} \\
b_{1} & b_{0} & b_{3} & -b_{2} \\
b_{2} & -b_{3} & b_{0} & b_{1} \\
b_{3} & b_{2} & -b_{1} & b_{0}
\end{array}\right]\left[\begin{array}{l}
a_{0} \\
a_{1} \\
a_{2} \\
a_{3}
\end{array}\right]=\left[\begin{array}{l}
a_{0} b_{0}-a_{1} b_{1}-a_{2} b_{2}-a_{3} b_{3} \\
a_{0} b_{1}+a_{1} b_{0}+a_{2} b_{3}-a_{3} b_{2} \\
a_{0} b_{2}-a_{1} b_{3}+a_{2} b_{0}+a_{3} b_{1} \\
a_{0} b_{3}+a_{1} b_{2}-a_{2} b_{1}+a_{3} b_{0}
\end{array}\right] .
$$

Thus,

$$
\begin{aligned}
a \mathrm{~b}= & \left(a_{0} b_{0}-a_{2} b_{2}-a_{1} b_{1}-a_{3} b_{3}\right)+\left(a_{0} b_{1}+a_{1} b_{0}+a_{2} b_{3}-a_{3} b_{2}\right) i_{1} \\
& +\left(a_{0} b_{2}-a_{1} b_{3}+a_{2} b_{0}+a_{3} b_{1}\right) i_{2}+\left(a_{0} b_{3}+a_{1} b_{2}-a_{2} b_{1}+a_{3} b_{0}\right) i_{3} .
\end{aligned}
$$

Is there any advantage in choosing specific matrix representation of a quaternion number in solving a problem? This is the third question, and it will be left for future work.

\section{Conclusion}

Each one of the four real matrices, $A_{1}$ (left matrix representation), $B_{1}$ (transpose matrix of $A_{1}$ ), $C_{1}$ (right matrix representation), and $D_{1}$ (transpose matrix of $C_{1}$ ), gives 23 distinct matrices, so precisely, there are 96 real matrices that represent a quaternion number where each one of them consists of three $4 \times 4$ skew-symmetric matrices plus the identity matrix.

\section{Data Availability}

No data were used to support the findings of the study.

\section{Conflicts of Interest}

The author declares no conflicts of interest.

\section{References}

[1] T.-Y. Lam, Hamilton's Quaternions, Handbook of Algebra, Amsterdam, The Netherlands, 2003.

[2] D. W. Lewis, "Quaternion algebras and the algebraic legacy of Hamilton's quaternions," Irish Mathematical Society, vol. 57, pp. 41-64, 2006.

[3] W. R. Hamilton, "On quaternions or a new system of imaginaries in algebra, philosophical magazine," 2000.

[4] R. Goldman, "Understanding quaternions," Graphical Models, vol. 73, no. 2, p. 21, 2011.

[5] J. Pujol, “On Hamilton's nearly-forgotten early work on the relation between rotations and quaternions and on the composition of rotations," American Mathematical Monthly, vol. 121, 2014. 
[6] A. van Weerden and S. Wepster, "A most gossiped about genius: Sir William Rowan Hamilton," BSHM Bulletin: Journal of the British Society for the History of Mathematics, vol. 33, no. 1, pp. 2-20, 2018.

[7] J. H. Conway and D. A. Smith, "On quaternions and octonions," AMC, vol. 10, p. 12, 2003.

[8] R. A. Reyment, K. G. Jöreskog, and L. F. Marcus, Applied Factor Analysis in the Natural Sciences, Cambridge University Press, London, UK, 1996.

[9] T. A. Whitelaw, Introduction to Linear Algebra, CRC Press, London, UK, 2nd edition, 1991.

[10] R. W. Farebrother, J. Groß, and S.-O. Troschke, "Matrix representation of quaternions," Linear Algebra and Its Applications, vol. 362, pp. 251-255, 2003. 